Max-Planck-Institut für demografische Forschung

Max Planck Institute for Demographic Research

Konrad-Zuse-Strasse 1 - D-18057 Rostock = Germany = Tel +49 (0) 3812081 - 0 - Fax +49 (0) 3812081 - 202 - www.demogr.mpg.de

MPIDR Technical Report TR 2018-002 I January 2018

\title{
R programs for splitting abridged \\ fertility data into a fine grid of ages using the quadratic optimization method
}

Anatoli I. Michalski I mpoctok@yandex.ru

Pavel Grigoriev I grigoriev@demogr.mpg.de

Vasily P. Gorlishchev I gpa15@yandex.ru

For additional material see www.demogr.mpg.de/tr

\footnotetext{
This technical report has been approved for release by: Dmitri A.Jdanov (jdanov@demogr.mpg.de), Head of the Laboratory of Demographic Data.

(C) Copyright is held by the authors.
} 


\title{
$\mathbf{R}$ programs for splitting abridged fertility data into a fine grid of ages using the quadratic optimization method
}

\author{
Anatoli I. Michalski (mpoctok@yandex.ru) \\ Pavel Grigoriev (grigoriev@demogr.mpg.de) \\ Vasily P. Gorlishchev (gpa15@yandex.ru)
}


Abstract. Occasionally, it is necessary to split aggregated fertility data into a fine grid of ages. In this technical report, we present an application of the quadratic optimization (QO) method for splitting abridged fertility data. The QO approach allows us to split not only age-specific fertility rates, but also birth counts in cases in which population exposures are available. Unlike the existing methods, the QO algorithm simultaneously satisfies five criteria of the 'ideal' disaggregation method: 1) shape - the estimated fertility curves should be plausible and smooth; 2) fit - the predicted values should be as close as possible to the observed values; 3) non-negativity - only positive values should be returned for the birth-order data; 4) balance - the estimated five-year age group totals should match the input data; and 5) parity - the balance by parity must be maintained. The examples provided in this report demonstrate the simplicity, flexibility, and high degree of effectiveness of the proposed technique. 


\section{Background}

The challenge of having to split aggregated fertility data into single years of age is often encountered by demographers. To tackle this problem, several reasonably effective disaggregation methods have been developed and tested (McNeil et. 1975; Smith, Hyndman, Wood, 2004; Liu, et al. 2011; Schmertmann 2012; Jasilioniene et al. 2012, Grigoriev and Jdanov, 2015). The need for disaggregation tends to arise when demographers are using historical data or data from developing countries that do not have functioning systems of vital registration. Splitting is often required for the purposes of data harmonization; i.e., for ensuring that the data are comparable across time and countries. The issue of splitting is particularly relevant in discussions about the maintenance of large international databases such as the Human Fertility Database (http://www.humanfertility.org) and the Human Fertility Collection (http://www.fertilitydata.org). Currently, the HFD has its own splitting protocol, the HFD method (Jasilioniene et al. 2012); while the HFC uses the Calibrated Spline (CS) estimator (Schmertmann, 2012) to disaggregate age-specific fertility rates (Grigorieva et al. 2015). Both methods work reasonably well, but they also have certain disadvantages (see Grigoriev et al. 2018). These challenges motivated us to develop a new method that can be universally applied to both the high-quality HFD data and the heterogeneous and noisy HFC data. Our new method simultaneously satisfies the following criteria:

1) Shape - the estimated fertility curves should be plausible and smooth;

2) Fit - the predicted values should be as close as possible to the observed values;

3) Non-negativity - only positive values should be returned;

4) Balance - the estimated five-year age group totals should match the input data; and

5) Parity - the balance by parity must be maintained.

To our knowledge, none of the existing methods fully meets the first four criteria, and no attempt has been made to extend the restrictions to criterion (5).

\section{Description of the algorithm}

The problem of splitting aggregated fertility data into single years of age is formulated as the reconstruction of a smooth curve, which satisfies criteria (1-5). The smoothness of the disaggregated age-specific fertility rates (ASFR) is determined by the quadratic function, which equals the sum of the squares of the second order divided differences for the ASFR, which have to be as small as possible. In this way, the problem of splitting aggregated fertility data is reduced to the problem of quadratic optimization under a set of linear constraints derived from criteria (2-5). 
Mathematically, the quadratic optimization problem for the age-specific fertility rate calculation can be formulated as

$$
\begin{aligned}
& \text { Minimize }\|F \times f r\|^{2} \quad \text { criterion (1) } \\
& \text { subject to } G \times f r-b=0 \quad, \quad \text { criterion (4) } \\
& f r_{j} \geq 0, j=1, \ldots, n . \quad \text { criterion (3) }
\end{aligned}
$$

Here, $n$ is the number of years for which the age-specific fertility rate is calculated, $f r$ is the vector of the one-year ASFR values, $\quad F$ is the $(n-2) \times n$ matrix for the calculation of the secondorder divided differences, $b$ is the vector of the observed number of births by $m$ five-year age groups, and $G$ is the $m \times n$ matrix of the number of women exposed to a birth event at a specific age in a specific five-year age group.

The quadratic optimization problem takes on a more complex form when both age- and parityspecific fertility rates have to be estimated. That is,

$$
\begin{aligned}
& \text { minimize } \quad\|F \times f r\|^{2}+\sum_{p=1}^{5}\left\|F \times f r^{p}\right\|^{2} \\
& \text { criterion (1) } \\
& \text { subject to } \quad G \times f r-b=0 \\
& \text { criterion (4) } \\
& G^{p} \times f r^{p}-b^{p}=0 \\
& p=1, \ldots, 5 \\
& \text { criterion (4) } \\
& E_{j} f r_{j}-\sum_{p=1}^{5} E_{j}^{p} \times f r_{j}^{p}=0 \\
& j=1, \ldots, n \\
& \text { criterion (5) } \\
& f r_{j} \geq 0 \\
& j=1, \ldots, n, \\
& \text { criterion (3) } \\
& f r_{j}^{p} \geq 0 \\
& j=1, \ldots, n, \quad p=1, \ldots, 5 \quad \text { criterion (3) }
\end{aligned}
$$

The new notations $f r^{p}$ are - the vectors of one-year ASFRs by parities 1,2,3,4, and 5+; $b^{p}$ - the vectors of the observed number of births by $m$ five-year age groups and by parities $1,2,3,4$, and $5+; G^{p}-m \times n$ matrixes of the number of women exposed to births at a specific age in a specific five-year age group and parities $1,2,3,4$, and $5+; E_{j}$ - the number of women in the $j$-th one-year age group exposed to births; and $E_{j}^{p}$ - the number of women in the $j$-th one-year age group who had already given birth to $p-1$ children. In the case of unconditional estimates for the age-specific fertility rate, all of the $G^{p}$ matrixes are equal to the $G$ matrix, and the vectors $E$ and $E^{p}$ are the rows of the $G$ matrix. 


\section{Requirements}

The scripts were tested using $R$ version 3.1. The application of the $Q O$ method requires the use of the 'quadprog' package, which must be installed from a standard $\mathrm{R}$ repository (CRAN).

\section{Usage}

The .zip file included in the technical report contains $R$ scripts and the data files used in the examples. R functions QOSplit.R and QOSplitPar. $R$ have to be loaded into an $R$ environment using the source command. A description of these functions is provided below.

\section{1. $R$ functions}

\subsubsection{QOSplit.R()}

This function splits the aggregated age-specific fertility rates (ASFRs) into single years of age using the quadratic optimization procedure.

Usage: QOSplit (Fx ,L, Agelnt)

The arguments are as follows:

$F x$ - the vector of the aggregated ASFRs to be split into single ages;

$L$ - the vector containing the lower limits of the age intervals (e.g., $L=10$ for age interval 10-14); and Agelnt - the vector containing the length of the age intervals.

All of the arguments should have the same length.

Example: QOSplit $(F x=c(0.00008,0.02655,0.10538,0.09983,0.05105,0.01904,0.00359$, 0.00033, 0.00000), $L=\operatorname{seq}(10,50,5)$, Agelnt=rep $(5,9))$

QOSplit.R returns the data frame in the following format: 


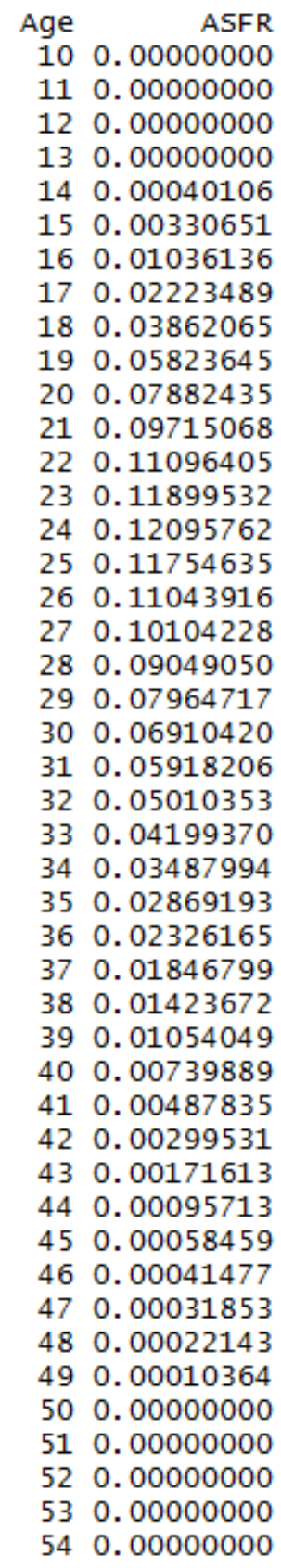

By default, the minimum and the maximum ages (here, 10 and 54) in the output correspond to the minimum and the maximum ages of the first (10-14) and the last (50-54) age intervals in the input data.

\subsubsection{QOSplitPar.R()}

This function splits the aggregated age- and parity-specific fertility data (either rates or births counts) into single years of age using a quadratic optimization procedure. In cases in which the population exposures by single year of age are not available, the function can only return the ASFRs. 
Usage: QOSplitPar<-function(CY,FX,Pop5,Pop1=NULL, L, Agelnt, Rates=TRUE)

The arguments are as follows:

CY - the country-year string (e.g., "AUT1984");

$F x$ - the data frame containing the aggregated birth counts to be split;

Pop5 - the vector of the population exposures (aggregated): length(Pop5)=length(Fx);

Pop1 - the vector of the population exposures by single year of age: length(Pop1)=length(AgeOutput) (Note: By default, the Pop1=NULL - data are not available.);

$L$-the vector containing the lower limits of the age intervals (e.g., $L=10$ for the age interval 10-14)

Agelnt - the vector containing the length of the age intervals; and

Rates - in response to the question of whether rates or birth counts should be returned, Rates=FALSE - birth counts should be returned, and otherwise rates should be returned (Note: By default, Rates=TRUE because Pop1=NULL).

Example:

QOSplitPar(CY="FIN2004", Fx=d5[,5:10],Pop5=d5\$Exposure,Pop1=NULL, $L=d 5 \$$ Age,Agelnt=d5\$Agelnt, Rates=TRUE)

QOSplitPar.R returns the data frame in the following format: 


\begin{tabular}{|c|c|c|c|c|c|c|c|c|}
\hline ge & ASFRO & ASFR1 & ASFR2 & ASFR3 & ASFR4 & ASFR 5P & ntryYear & Diff \\
\hline 12 & 0.00000000 & 0.00000000 & 0.00000000 & 0.00000000 & 0.00000000 & 0.00000000 & FIN2004 & 0.00000 \\
\hline 13 & 0.00000000 & 0.00000000 & 0.00000000 & 0.00000000 & 0.00000000 & 0.00000000 & FIN2004 & 0.00000 \\
\hline 14 & 0.00012292 & 0.00012292 & 0.00000000 & 0.00000000 & 0.00000000 & 0.00000000 & FIN2004 & 0.00000 \\
\hline 15 & 0.00168088 & 0.00171299 & 0.00000000 & 0.00000000 & 0.00000000 & 0.00000000 & FIN2004 & -0.000 \\
\hline 16 & 0.00463514 & 0.00466725 & 0.00000000 & 0.00000000 & 0.00000000 & 0.00000000 & FIN2004 & -0.000 \\
\hline 17 & 0.00896009 & 0.00886673 & 0.00009336 & 0.00000000 & 0.00000000 & 0.00000000 & FIN2004 & 0.000 \\
\hline 18 & 0.01497605 & 0.01384366 & 0.00109648 & 0.00000000 & 0.00000381 & 0.00000000 & FIN2004 & \\
\hline & 0.02289256 & 0.01923820 & .00336921 & 0.00022474 & 0.00002830 & 0.00000000 & FIN2004 & 0.00 \\
\hline 20 & 0.03274254 & 0.02486426 & .00688077 & 0.00092371 & 0.00008883 & 0.00000000 & FIN2004 & -0.000 \\
\hline 21 & 0.04431083 & 0.03078099 & 0.01128111 & 0.00200885 & 0.00027071 & 0.00000000 & FIN2004 & -0.00 \\
\hline 22 & 0.05722504 & 03690366 & 0.01625608 & 00341569 & 0.00062999 & 0.000025 & $=$ IN 2004 & \\
\hline 23 & 0.07097391 & 0.04298532 & 0.02150905 & 0.00508500 & 0.00117509 & 0.0001 & FIN2004 & 0 \\
\hline 24 & 0.08488892 & 0.04863515 & 0.02677927 & 0.00698116 & 0.00188521 & 0.00057731 & FIN2004 & 0 \\
\hline 2 & 0.09814 & 0.05331850 & 0.03184188 & 0.00909216 & 0.00271028 & 0.001157 & FIN2004 & 0 \\
\hline & 0.1097 & 0.05635 & 0.03650 & 0.01143 & 0.00357 & 0.00 & FIN2004 & \\
\hline 27 & 0. & 87 & 6 & 139 & 0 . & 0 . & FIN & 0 \\
\hline 28 & 0.12508 & 0.05615939 & ג קרי & 0.01650449 & 0.00517 & 0.003469 & FIN2004 & -0.00 \\
\hline 29 & 0.127819 & 0.05292544 & 0.04591159 & 0.01892 & 0.00583 & 0.0042 & FIN2004 & -0 . \\
\hline 3 & 0.12692 & 0.04805 & 0466 & & 0 . & & 04 & \\
\hline 31 & 0.1224 & 4223964 & & 0.02222 & 0.00681 & 0.00 & FIN2004 & -0 \\
\hline 3 & 0.114516 & 0.03603456 & 0.04297412 & 0.02258 & 0.00711 & 0.00 & FIN2004 & 0 \\
\hline 33 & 0.103723 & 0.02989062 & 0.03861743 & 0.02194878 & 0.00727990 & 0.0059 & FIN2004 & 0 . \\
\hline 34 & 0.09077 & 0.02413831 & 0.03299 & 00203 & 0. & 0.00 & FIN & \\
\hline 3 & 0.076646 & 0.01899098 & 0.02667907 & 0.01809 & 0 . & 0.00 & FIN & 0 \\
\hline 3 & 0.062571 & 0.01454481 & 0.020491 & 0.01540 & 0.00665 & 0.00 & FIN & \\
\hline 37 & 0.049473 & 0.01084595 & 0.01500616 & 0.01258647 & 0.00599630 & 0.0050 & FIN2004 & 0.00 \\
\hline 38 & 0.037968 & 0.00789047 & 0.01056 & 0. & 0. & 0 . & FIN & \\
\hline 3 & 0.0 & 0.00562444 & 0.00729367 & 0. & 0 . & 0 . & FIN & -0 \\
\hline 40 & 0.02067 & 0.003943 & 0.00506566 & 0.005248 & 0.00306 & 0.00337 & FIN & -0 \\
\hline 41 & 0.014601 & 0.00269460 & 0.00353701 & 0.00351929 & 0.00209 & 0.0027 & FIN2004 & -0.00 \\
\hline & 0.00987 & 0.00175819 & 0.00244 & 0.00220 & 0 . & 0 . & FIN & \\
\hline 4 & 0.006280 & 0.00105151 & 0.0016074 & 0.00127797 & 0.000724 & 0.00161 & FIN & 0 \\
\hline 44 & 0.003640 & 0.0005269 & 0.0009310 & 0.000695 & 0.000367 & 0.00110 & FIN & 0 \\
\hline 45 & 0.001817 & 0.00017243 & 0.0004031 & 0.000369 & 0.000182 & 0.00066 & FIN2004 & 0 \\
\hline 46 & 0.000713 & 0.00001129 & 0.00009 & 0.00018 & 0 . & 0 . & FIN & \\
\hline 47 & 0.000171 & 0.00000000 & 0.00000000 & 0.00007620 & 0.000024 & 0.00009 & FIN2004 & -0.000 \\
\hline 48 & 0.0000000 & 0.00000000 & 0.0000000 & 0.0000240 & 0.000001 & 0.00000 & FIN2004 & -0 \\
\hline 49 & 0.00000000 & 0.00000000 & 0.00000000 & 0.000004 & 0.000000 & 0.0000 & FIN2004 & \\
\hline 50 & 0.0000155 & 0.00000000 & 0.00000000 & 0.000000 & 0003 & 0.00001 & FIN2004 & \\
\hline 51 & 0.0000299 & 0.00000000 & 0.00000000 & 0.00000000 & 0.00000739 & 0.0000224 & FIN2004 & 0.00 \\
\hline$-\pi$ & 0.000033 & 0.00000000 & 0.00000000 & 0.0000000 & 0.0000084 & 0.000024 & FIN2004 & 0.00 \\
\hline & 0.000021 & 0.00000000 & 0.0000000 & 0.0000000 & 0.000005 & 0.00001 & FIN2004 & \\
\hline & 0.000000 & 0.00000000 & 0.0000000 & 0.0000000 & 0.0000000 & 0.00000000 & FIN2004 & 0.0 \\
\hline
\end{tabular}

Columns ASFR0, ASFR1, ASFR2, ASFR3, ASFR4, and ASFR5P refer to the total (0) age-specific fertility rate, and to the ASFRs by parities 1,2,3,4, and 5+, respectively. Variable Diff indicates a small difference between ASFR0 and the sum of ASFR1-ASFR5P.

The output containing birth counts has the same structure.

QOSplitPar(CY="FIN2004",FX=d5[,5:10],Pop5=d5\$Exposure,Pop1=d1\$Exposure, $L=d 5 \$ A g e$, Agelnt=d5\$Agelnt, Rates=FALSE) 


\begin{tabular}{|c|c|c|c|c|c|c|c|c|}
\hline ge & BO & B1 & B2 & B3 & B4 & $B 5 P$ & ntryYear & Diff \\
\hline 12 & 0.00 & 0.00 & 0.00 & 0.00 & 0.00 & 0.00 & FIN2004 & 0.00 \\
\hline 13 & 0.00 & 0.00 & 0.00 & 0.00 & 0.00 & 0.00 & FIN2004 & 0.00 \\
\hline 14 & 3.97 & 3.97 & 0.00 & 0.00 & 0.00 & 0.00 & FIN2004 & 0.00 \\
\hline 15 & 53.04 & 54.06 & 0.00 & 0.00 & 0.00 & 0.00 & FIN2004 & -1.02 \\
\hline 16 & 143.29 & 144.28 & 0.00 & 0.00 & 0.00 & 0.00 & FIN2004 & -0 . \\
\hline 17 & 269.70 & 266.89 & 2.81 & 0.00 & 0.00 & 0.00 & FIN2004 & \\
\hline 18 & 464.36 & 429.24 & 34.00 & 0.00 & 0.12 & 0.00 & FIN2004 & 1.00 \\
\hline 19 & 736.14 & 618.63 & 108.34 & 7.23 & 0.91 & 0.00 & FIN2004 & 1.03 \\
\hline 20 & 1081.99 & 821.65 & 227.38 & 30.52 & 2.94 & 0.00 & FIN2004 & -0.50 \\
\hline 21 & 1472.33 & 1022.77 & 374.84 & 66.75 & 8.99 & 0.00 & FIN2004 & -1.0 \\
\hline 22 & 1855.53 & 1196.60 & 527.11 & 110.75 & 20.43 & 0.83 & FIN2004 & \\
\hline 23 & 2249.15 & 1362.19 & 681.62 & 161.14 & 37.24 & 6.29 & FIN2004 & \\
\hline 24 & 2702.97 & 1548.60 & 852.69 & 222.29 & 60.03 & 18.38 & 004 & \\
\hline 25 & 3155.60 & 1714.33 & 1023.80 & 292.34 & 87.14 & 37.23 & 004 & \\
\hline 26 & 3523.61 & 1809.34 & 1172.11 & 366.99 & 114.70 & 60.17 & FIN2004 & 0 . \\
\hline 27 & 3888.03 & 1874.80 & 1326.29 & 455.81 & 144.17 & 87.10 & FIN2004 & -0.1 \\
\hline 28 & 4070.70 & 1827.59 & 1425.09 & 537.10 & 168.45 & 112.91 & FIN2004 & -0. \\
\hline 29 & 4026.63 & 1667.28 & 1446.33 & 596.14 & 183.97 & 133.41 & FIN2004 & -0.5 \\
\hline 30 & 3743.00 & 1417.17 & 1375.63 & 617.50 & 188.31 & 144.74 & FIN2004 & -0 \\
\hline 31 & 3445.39 & 1188.85 & 1286.18 & 625.55 & 191.78 & 153.18 & 2004 & -0 \\
\hline 32 & 3408.00 & 1072.39 & 1278.91 & 672.10 & 211.81 & 172.76 & 04 & 0. \\
\hline 33 & 3202.95 & 923.01 & 1192.49 & 677.77 & 224.80 & 184.68 & 004 & \\
\hline 34 & 2869.14 & 762.96 & 1042.76 & 644.38 & 230.09 & 188. & 004 & \\
\hline 35 & 2565.04 & 635.55 & 892.84 & 605.59 & 237.11 & 193. & 04 & \\
\hline 36 & 2232.32 & 518.90 & 731.07 & 549.58 & 237.56 & 195. & 04 & 0 . \\
\hline 37 & 1781.12 & 390.47 & 540.24 & 453.13 & 215.87 & 181.39 & 04 & 0 . \\
\hline 38 & 1375.56 & 285.86 & 382.88 & 357.12 & 185.76 & 164.11 & 004 & -0.1 \\
\hline 39 & 1047.48 & 207.67 & 269.31 & 272.39 & 151.98 & 146.48 & 004 & -0.3 \\
\hline 40 & 774.03 & 147.61 & 189.60 & 196.47 & 114.54 & 126.25 & FIN2004 & -0.4 \\
\hline 41 & 548.34 & 101.19 & 132.83 & 132.16 & 78.55 & 103.97 & FIN2004 & -0.3 \\
\hline 42 & 369.16 & 65.73 & 91.37 & 82.32 & 48.55 & 81.31 & FIN2004 & -0.1 \\
\hline 43 & 233.77 & 39.14 & 59.83 & 47.57 & 26.95 & 60.03 & 2004 & 0 . \\
\hline 44 & 136.06 & 19.69 & 34.80 & 25.99 & 13.72 & 41. & 04 & 0 . \\
\hline 45 & 66. & 6.32 & 14.78 & 13. & 6.68 & 24 & 04 & 0.9 \\
\hline 46 & 26.60 & 0.42 & 3.56 & 6. & 2.99 & 11 & 04 & 0 . \\
\hline 47 & 6.59 & 0.00 & 0.00 & 2.92 & 0.93 & 3 & FIN2004 & -0. \\
\hline 48 & 0.00 & 0.00 & 0.00 & 0.94 & 0.07 & 0 . & FIN2004 & -1.0 \\
\hline 49 & 0.00 & 0.00 & 0.00 & 0.16 & 0.00 & 0 . & FIN2004 & -0.1 \\
\hline 50 & 0.61 & 0.00 & 0.00 & 0.00 & 0.14 & 0. & 004 & \\
\hline 51 & 1.19 & 0.00 & 0.00 & 0.00 & 0.29 & 0.89 & FIN2004 & 0. \\
\hline 52 & 1.30 & 0.00 & 0.00 & 0.00 & 0.33 & 0.98 & FIN2004 & -0.01 \\
\hline 53 & 0.87 & 0.00 & 0.00 & 0.00 & 0.23 & 0.65 & FIN2004 & -0.0 \\
\hline 54 & 0.00 & 0.00 & 0.00 & 0.00 & 0.00 & 0.00 & FIN2004 & 0.0 \\
\hline
\end{tabular}

\subsection{Format of the input data}

The input data appear in the form of .csv (comma separated values) files, with the first line containing the variable names (header). Five data files are used in the examples:

- 'HFD abridged data 7 groups.csv',

- 'HFD abridged data 9 groups.csv',

- 'HFD abridged data 7 groups BO.csv',

- 'HFD abridged data 9 groups BO.csv', and

- 'BirthsExposASFR1x1_BO.csv'. 
Files (1) and (2) refer to the input data with seven and nine age groups, respectively. These files have to be used by QOSplit.R.

Fragment of 'HFD abridged data 7 groups.csv':

\begin{tabular}{|c|c|c|c|c|c|c|c|c|}
\hline Age & countryyear & code & Year & Births & Exposure & ASFR & AgeGr Labe1 & AgeInt \\
\hline 15 & AUT1951 & AUT & 1951 & 7350 & 215582.54 & 0.0340936701089 & $15-19$ & \\
\hline 20 & AUT1951 & AUT & 1951 & 27775 & 242049.79 & 0.1147491183529 & $20-24$ & \\
\hline 25 & AUT1951 & AUT & 1951 & 32974 & 291065.68 & 0.1132871453618 & $25-29$ & \\
\hline 30 & AUT1951 & AUT & 1951 & 17917 & 217312.90 & 0.0824479356725 & $30-34$ & \\
\hline 35 & AUT1951 & AUT & 1951 & 11638 & 257812.81 & 0.0451412790544 & $35-39$ & \\
\hline 40 & AUT1951 & AUT & 1951 & 4702 & 293646.64 & 0.0160124427101 & $40-44$ & \\
\hline 45 & AUT1951 & AUT & 1951 & 393 & 554677.92 & 0.0007085192791 & $45-49$ & \\
\hline 15 & AUT1952 & AUT & 1952 & 7184 & 208671.92 & 0.0344272482853 & $15-19$ & \\
\hline 20 & AUT1952 & AUT & 1952 & 27770 & 234527.53 & 0.1184082738602 & $20-24$ & \\
\hline 25 & AUT1952 & AUT & 1952 & 32427 & 280115.63 & 0.1157629083390 & $25-29$ & \\
\hline
\end{tabular}

Fragment of 'HFD abridged data 9 groups.csv':

\begin{tabular}{|c|c|c|c|c|c|c|c|c|}
\hline Age & Countryyear & code & Year & Births & Exposure & ASFR & AgeGr Labe 1 & AgeInt \\
\hline 10 & AUT1951 & AUT & 1951 & 15.00 & 140071.63 & 0.00010708806630 & $10-14$ & 5 \\
\hline 15 & AUT1951 & AUT & 1951 & 7350.00 & 215582.54 & 0.03409367010891 & $15-19$ & 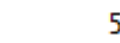 \\
\hline 20 & AUT1951 & AUT & 1951 & 27775.00 & 242049.79 & 0.11474911835288 & $20-24$ & \\
\hline 25 & AUT1951 & AUT & 1951 & 32974.00 & 291065.68 & 0.11328714536183 & $25-29$ & \\
\hline 30 & AUT1951 & AUT & 1951 & 17917.00 & 217312.90 & 0.08244793567248 & $30-34$ & \\
\hline 35 & AUT1951 & AUT & 1951 & 11638.00 & 257812.81 & 0.04514127905437 & $35-39$ & \\
\hline 40 & AUT1951 & AUT & 1951 & 4702.00 & 293646.64 & 0.01601244271005 & $40-44$ & \\
\hline 45 & AUT1951 & AUT & 1951 & 379.59 & 287396.02 & 0.00132079073329 & $45-49$ & \\
\hline 50 & AUT1951 & AUT & 1951 & 13.41 & 267281.90 & 0.00005017174751 & $50-54$ & \\
\hline 10 & AUT1952 & AUT & 1952 & 18.00 & 163855.71 & 0.00010985274788 & $10-14$ & \\
\hline
\end{tabular}

Note: For testing purposes, these data files include more variables than are actually needed. Only four variables are used by function QOSplit.R: Age, CountryYear, ASFR, and Agelnt.

Files (3) and (4) refer to the parity-specific fertility data aggregated into seven and nine age groups. These files have to be used by QOSplitPar.R

Fragment of 'HFD abridged data 7 groups BO.csv': 


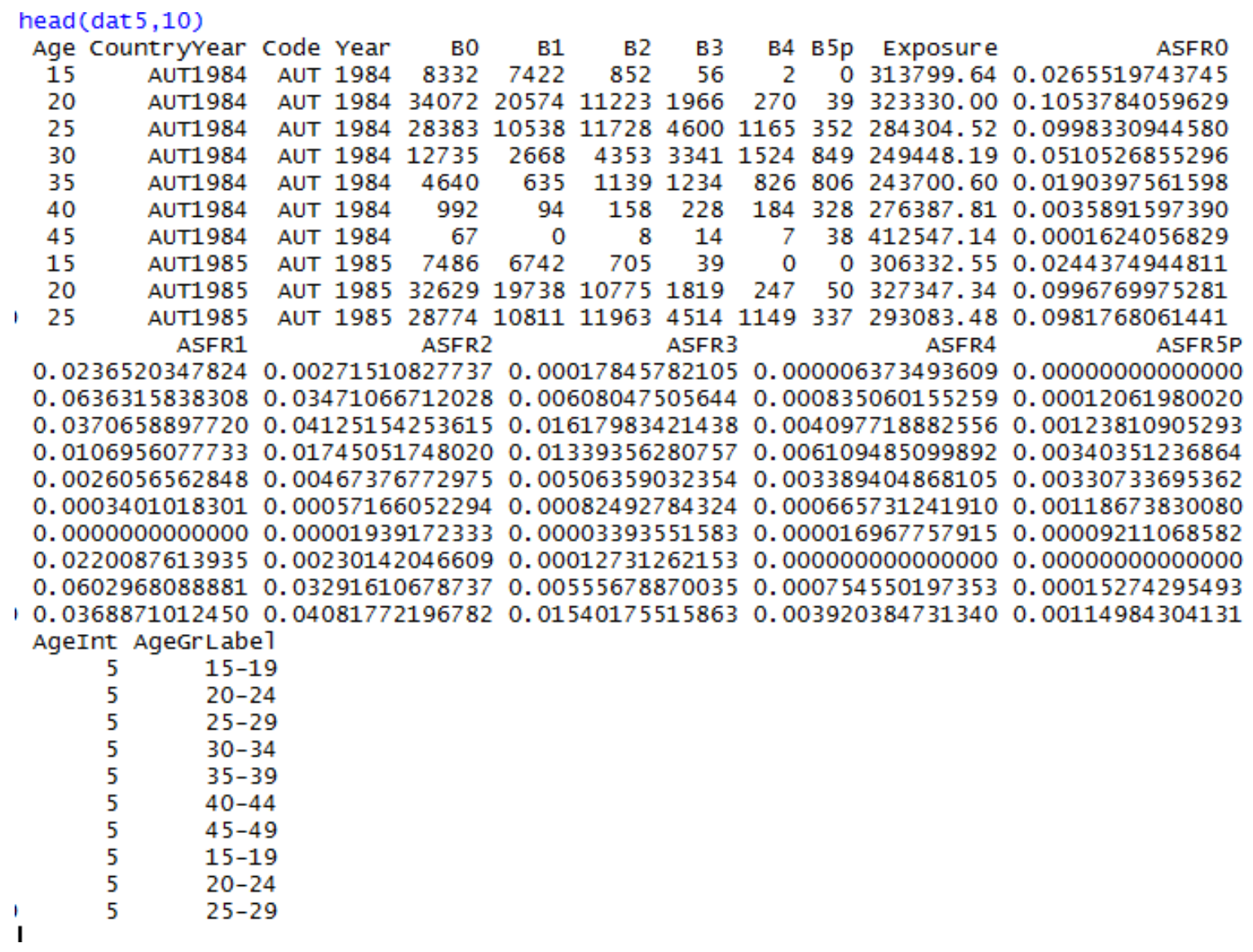

Fragment of 'HFD abridged data 9 groups BO.csv': 


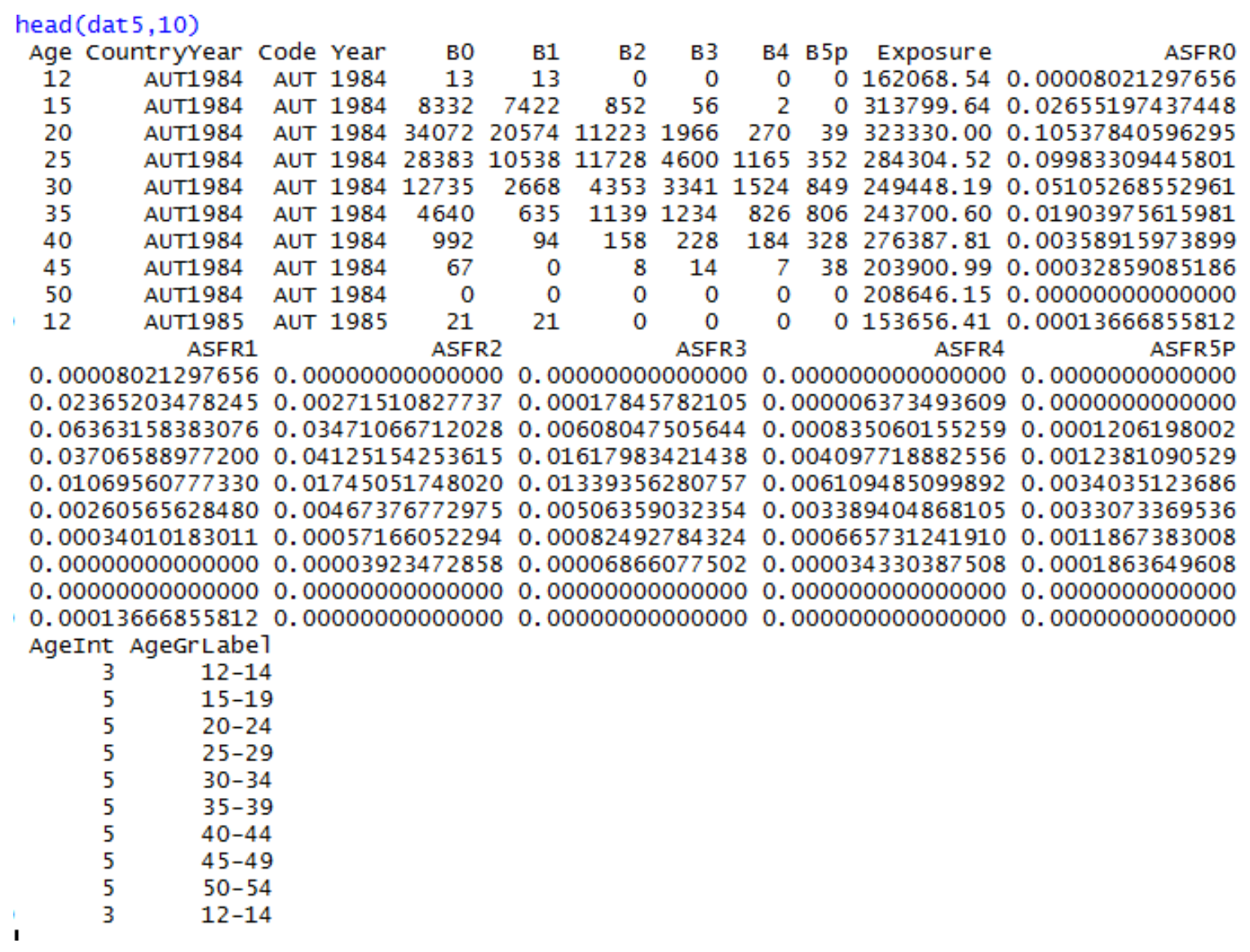

Note: For testing purposes, these data files include more variables than are actually needed. The following variables are used by function QOSplitPar.R: Age, CountryYear, B0, B1, B2, B3, B4, B5p, Exposure, and Agelnt.

Both of the $B O$ files were generated from the original birth counts and the population exposures by single year of age ('BirthsExposASFR1x1_BO.csv'). This file provides the information on the population exposures (argument Pop1 of the function QOSplitPar.R). Additionally, this file contains the original fertility estimates we used to assess the model fit.

Fragment of 'BirthsExposASFR1x1_BO.csv': 


\begin{tabular}{|c|c|c|c|c|c|c|c|c|c|c|c|c|}
\hline countryYear & code & Year & Age & Agegroup & $\mathrm{BO}$ & $\mathrm{B} 1$ & $\mathrm{~B} 2$ & B3 & B4 & B $5 p$ & Exposure & ASFRO \\
\hline AUT1984 & AUT & 1984 & 12 & $10-14$ & 0 & 0 & 0 & 0 & 0 & 0 & 51383.50 & 0.000000000 \\
\hline AUT1984 & AUT & 1984 & 13 & $10-14$ & 3 & 3 & 0 & 0 & 0 & 0 & 53807.85 & 0.00005580 \\
\hline AUT1984 & AUT & 1984 & 14 & $10-14$ & 10 & 10 & 0 & 0 & 0 & 0 & 56877.19 & 0.00017581 \\
\hline AUT1984 & AUT & 1984 & 15 & $15-19$ & 96 & 94 & 2 & 0 & 0 & 0 & 60561.21 & 0.00158517 \\
\hline AUT1984 & AUT & 1984 & 16 & $15-19$ & 405 & 389 & 15 & 1 & 0 & 0 & 61930.79 & 0.00653955 \\
\hline AUT1984 & AUT & 1984 & 17 & $15-19$ & 1243 & 1183 & 58 & 2 & 0 & 0 & 62726.76 & 0.01981610 \\
\hline AUT1984 & AUT & 1984 & 18 & $15-19$ & 2417 & 2217 & 190 & 9 & 1 & 0 & 63175.63 & 0.03825842 \\
\hline AUT1984 & AUT & 1984 & 19 & $15-19$ & 4171 & 3539 & 587 & 44 & 1 & 0 & 65405.25 & 0.06377163 \\
\hline AUT1 & AUT & 1984 & 20 & $20-24$ & 5688 & 4422 & 1161 & 99 & 6 & 0 & 66104.74 & 0.08604526 \\
\hline AUT1984 & AUT & 1984 & 21 & $20-24$ & 6581 & 4415 & 1908 & 222 & 35 & 1 & 66588.15 & 0.09883139 \\
\hline
\end{tabular}

$\begin{array}{lllllll}0.000000000 & 0.000000000 & 0.000000000 & 0.000000000 & 0.000000\end{array}$

$\begin{array}{llllllll}0.000055800 & 0.000000000 & 0.000000000 & 0.000000000 & 0.000000\end{array}$

$\begin{array}{llllllll}0.000175817 & 0.000000000 & 0.000000000 & 0.000000000 & 0.000000\end{array}$

$\begin{array}{lllllll}0.001552149 & 0.000033000 & 0.000000000 & 0.000000000 & 0.000000\end{array}$

$\begin{array}{llllllll}0.006281205 & 0.000242206 & 0.000016100 & 0.000000000 & 0.000000\end{array}$

$\begin{array}{lllllll}0.018859574 & 0.000924645 & 0.000031900 & 0.000000000 & 0.000000\end{array}$

$\begin{array}{llllllll}0.035092646 & 0.003007489 & 0.000142460 & 0.000015800 & 0.000000\end{array}$

$\begin{array}{llllllll}0.054108806 & 0.008974815 & 0.000672729 & 0.000015300 & 0.000000\end{array}$

$\begin{array}{llllllll}0.066893842 & 0.017563037 & 0.001497623 & 0.000090800 & 0.000000\end{array}$

$\begin{array}{lllllll}0.066303088 & 0.028653747 & 0.003333927 & 0.000525619 & 0.000015\end{array}$

\section{Examples}

The R scripts ExamplesQOSplit.R and ExamplesQOSplitPar.R included in the .zip file contain various examples in which the functions QOSplit and QOSplitPar are used. As the scripts are accompanied by detailed comments, even inexperienced $\mathrm{R}$ users should find the QO method fairly simple to use.

\section{References}

Grigoriev P, Jdanov DA (2015). Splitting abridged fertility data using different interpolation methods: is there the optimal solution? Presentation at the $80^{\text {th }}$ PAA Meeting, San Diego, USA.

http://www.humanfertility.org/Docs/paa/Grigoriev Jdanov.pdf

Grigoriev P, Michalski AI, Gorlishchev VP, Jdanov DA, Shkolnikov VM (2018). New methods for estimating detailed fertility schedules from abridged data. MPIDR Working Paper WP-2018-001. Rostock. Available at http://www.demogr.mpg.de/papers/working/wp-2018-001.pdf

Grigorieva O, Jasilioniene A, Jdanov DA, Grigoriev P, Sobotka T, Zeman K, Shkolnikov VM (2015). Methods Protocol for the Human Fertility Collection. Available at: http://www.fertilitydata.org/docs/methods.pdf

Jasilioniene A, Jdanov DA, Sobotka T, Andreev EM, Zeman K, Nash EJ, and Shkolnikov VM. (with contributions of Goldstein J, Philipov D, and Rodriguez G) (2012). Methods Protocol for the Human Fertility Database. Available at: http://www.humanfertility.org

Liu Y, Gerland P, Spoorenberg T, Kantorova V, Andreev K (2011). Graduation methods to derive age-specific fertility rates from abridged data: a comparison of 10 methods using HFD data. Presentation at the First Human Fertility Database Symposium, Max Planck Institute for Demographic Research, Rostock, Nov 2011. http://www.humanfertility.org/Docs/Symposium/Liu-Gerland\%20et\%20al.pdf

McNeil DR, Trussell TJ, Turner JC (1977). Spline interpolation of demographic data. Demography 14(2): 245-252.

Schmertmann C (2012). Calibrated spline estimation of detailed fertility schedules from abridged data. MPIDR Working Paper WP-2012-022. Rostock.

Smith L, Hyndman R, Wood S (2004). Spline interpolation for demographic variables: the monotonicity problem. Journal of Population Research 21 (1), pp. 95-97.

Williams HP. (2013). Model Building in Mathematical Programming. Chichester: John Wiley \& Sons. 